\title{
In Vivo Mapping of Fiber Pathways in the Rhesus Monkey Brain
}

\author{
Sabine Hofer ${ }^{*}, 1,2$ and Jens Frahm $^{1}$ \\ ${ }^{1}$ Biomedizinische NMR Forschungs GmbH am Max-Planck-Institut für biophysikalische Chemie, 37070 Göttingen, \\ Germany \\ ${ }^{2}$ Bernstein Center for Computational Neuroscience, 37073 Göttingen, Germany
}

\begin{abstract}
The study of complex fiber systems in relation to the cognitive abilities of humans is a long-standing challenge for neuroscientists. With the development of diffusion tensor imaging (DTI) it is now possible to visualize large fiber bundles non-invasively. The existing knowledge of the white matter architecture largely stems from either lesion studies of human patients or, in more detail, tracer injection studies of non-human primates. Hence, it seems mandatory to compare DTI results with histochemical findings obtained for the same species. Using a geometrically undistorted DTI technique and fiber tractography, we examined the fiber anatomy of the macaque brain in vivo and related the results to fiber pathways previously identified in monkeys with conventional tract tracing. The approach identified multiple fiber tracts including the main association and projection pathways as well as fibers of the limbic system, commissural system, optic system, and cerebellar system. In conclusion, in vivo fiber tractography based on current-state DTI allows for a comprehensive analysis of major fiber pathways in the intact macaque brain.
\end{abstract}

Keywords: MRI, diffusion tensor imaging, fiber tractography, macaque brain.

\section{INTRODUCTION}

Cognitive and behavioral processes commonly involve a variety of cortical systems which are interwoven by multiple neuronal networks. A proper anatomical characterization of the fiber architecture underlying these networks is an important step toward the understanding of higher cerebral functions. Since the end of the $19^{\text {th }}$ century, techniques have been developed to examine the route of particular fiber bundles in different species. A popular strategy for studying fiber pathways in non-human primates is to apply a neuronal tracer such as horseradish peroxidase or lucifer yellow to single fibers or multi-fiber systems in vivo or in vitro. Active intraneuronal transport or passive diffusion spread the marker molecule within the neurons up to the fine ramifications of axons and dendrites, and thus allow for a detailed anatomical delineation of the target neurons (reviewed in [1]). Of course, tracer methods are invasive and therefore not suitable for healthy humans in vivo. At the end of the $20^{\text {th }}$ century, the development of diffusion-weighted MRI to diffusion tensor imaging (DTI) and its combination with tract tracing techniques $[2,3]$ for the first time visualized white matter fasciculi non-invasively in the living human brain (e.g., see $[4,5])$. In fact, far beyond the possibilities of conventional tracing techniques, DTI-based fiber tractography yields three-dimensional reconstructions of distinct fiber bundles in close correspondence to the individual brain anatomy - important factors or even prerequisites for applications in clinical neurology. On the other hand, a potential limitation of human DTI studies is the lack of validation. So far, DTIderived fiber tracts predominantly rely on comparisons with data gathered from dissections, myelin stains, and lesion studies $[6,7]$. As these human studies could not provide any

*Address correspondence to this author at the Biomedizinische NMR Forschungs GmbH, D-37070 Göttingen, Germany; Tel: +49-551-201-1735; Fax:+49-551-201-1307; E-mail: shofer1@gwdg.de details about the origin or termination of specific fiber bundles, most insights into the anatomy of respective pathways are still based on tracer injection studies of the macaque brain (as reviewed in [8]). In order to better link the in vivo identification of complex fiber pathways to conventional anatomical studies, and because the macaque brain is the best known primate brain in histological terms, we performed DTI and fiber tractography of the cerebral white matter of rhesus monkeys.

Technically, DTI exploits differences in the selfdiffusivity of water molecules that are caused by differences in the cellular microstructure of the brain tissue. While in large fluid-filled regions such as the ventricular spaces the self-diffusivity of water is equal in all directions, this is not the case in tissue. In particular, in white matter the water diffusivity is much less hindered along the fiber direction than perpendicular to the myelinated axon. Therefore, if axon bundles yield coherently arranged structures that scale to an image voxel, the diffusion properties become directionally dependent. Such anisotropic diffusion properties can be mathematically represented by a tensor, where the principle axis of the corresponding diffusion ellipsoid coincides with the direction of the greatest diffusion coefficient. This direction is then equated with the orientation of the underlying fiber bundle. In other words, the diffusion anisotropy reflects the degree to which diffusion is preferred along one direction relative to other directions.

Up to now, DTI-based fiber tracking has mainly been performed in humans. This is because of the relatively large brain volume in comparison to that of other primates or even smaller animals such as rodents (e.g., see [9]). A practical limitation is the achievable resolution and signal-to-noise ratio which may critically affect the determination of the major eigenvector and therefore lead to cumulative directional errors in the estimated fiber tracts. Here, we studied white matter fasciculi of the rhesus monkey brain in vivo at a 
relatively high magnetic field strength and with use of a most suitable radiofrequency coil originally designed for human shoulder imaging. In addition, we applied a geometrically undistorted DTI technique that avoids any sensitivity to susceptibility differences $[10,11]$. In contrast to the more often used echo-planar imaging (EPI) technique, STEAM sequences acquire stimulated echoes which are generated by the application of radiofrequency pulses. This kind of echo formation compensates for any influences of magnetic field inhomogeneities on the signal intensity and accuracy of spatial encoding. On the other hand, inhomogeneities affect MRI signals which are generated by reversal of a magnetic field gradient. Respective signal losses and geometric distortions most prominently accumulate in EPI sequences which exclusively rely on gradient echoes.

With reference to existing histochemical data, the optimized approach applied here allowed us to identify and visualize association and projection fibers as well as fiber bundles of the commissural, limbic, cerebellar, and optic system in a geometrically and anatomically correct manner.

\section{MATERIALS AND METHODS}

\section{Animals and MRI Data Acquisition}

For MRI, four male rhesus macaque (Macaca mulatta, 4 years) were premedicated using $0.01 \mathrm{mg} \mathrm{kg}^{-1}$ atropin. After induction by intramuscular injection of $15 \mathrm{mg} \mathrm{kg}^{-1}$ ketamine, general anesthesia was maintained by continuous intravenous propofol (10-15 mg kg-1). While in supine position, the animal head was centered within the concavity of a 4channel small shoulder coil using a home-made acrylic head holder (also described in [12]). Animal experiments were approved by national animal welfare authorities, LAVES, Braunschweig, Germany (reference no. 509.42502/0807.02).

Several animal MRI studies were performed to optimize the experimental conditions. Although similar findings were obtained in all monkeys, the final fiber reconstructions demonstrated here originate from a single animal. The ability of in vivo DTI to comprehensively map the white matter architecture of individual brains is a key advantage and a prerequisite for clinical applications.

MRI studies were conducted at 2.9 T (Tim Trio, Siemens Medical Solutions, Erlangen, Germany). Anatomic images were obtained using a $T_{1}$-weighted $3 \mathrm{D}$ fast low angle shot (FLASH) MRI sequence (repetition time TR $=12.3 \mathrm{~ms}$, echo time $\mathrm{TE}=4.9 \mathrm{~ms}$, flip angle $15^{\circ}$ ) yielding an isotropic spatial resolution of $0.5 \mathrm{~mm}$. Diffusion tensor imaging was performed at $1.5 \mathrm{~mm}$ isotropic resolution using diffusionweighted single-shot stimulated echo acquisition mode (STEAM) MRI sequences in combination with a projection onto convex subjects (POCS) reconstruction algorithm [11]. Because of the insensitivity of STEAM MRI acquisitions to magnetic field inhomogeneities, the undistorted STEAM images as well as the resulting fiber tracts are spatially congruent to the anatomical images. Without the need for a coregistration or alignment technique which are usually accompanied by a certain degree of blurring, the present work allowed for a direct digital superposition of corresponding data matrices.
Multislice cross-sectional images covering the whole brain (section thickness $1.5 \mathrm{~mm}$ ) were acquired along a horizontal orientation parallel to the anterior-posterior commissure (36 contiguous sections) covering a $192 \times 108 \mathrm{~mm}^{2}$ field-of-view by a $128 \times 72$ acquisition matrix. The highspeed STEAM imaging part of the sequence used TR $=7.8$ $\mathrm{ms}, \mathrm{TE}_{\mathrm{STE}}=8.6 \mathrm{~ms}$, and variable flip angles for generating 45 stimulated echoes (5/8 partial Fourier encoding). The diffusion-encoding spin-echo module comprised 24 gradient directions. The gradient strength corresponded to a $b$ value of $1000 \mathrm{~s} \mathrm{~mm}^{-2}$ and led to an echo time of $52.4 \mathrm{~ms}$ for the spin-echo preparation period required for diffusion encoding. Together with an additional measurement without diffusion encoding, each diffusion tensor was determined from a total of 25 scans. For an overall repetition time of TR $=15.576 \mathrm{~ms}$ and the recording of four averages the total measurement time was $28 \mathrm{~min}$.

\section{Fiber Tractography}

Before calculation of the diffusion tensor the diffusionweighted MRI data sets were interpolated to $0.75 \mathrm{~mm}$ isotropic resolution (half of the original linear voxel dimension or $1 / 8$ th of the voxel size). Estimates of axonal projections were computed by the fiber assignment by continuous tracking (FACT) algorithm [3] and tensorlines algorithm [13] using 9 seed points per image voxel, that is one central position complemented by two points half way on either side of each of the four spatial diagonals. The DTI analysis relied on software developed in house [14] and was previously applied to map the topography of transcallosal fiber connections in humans and monkeys $[5,12]$.

Tracking terminated when the fractional anisotropy (FA) was lower than 0.15 or the main diffusion directions in consecutive steps differed by more than $20^{\circ}-60^{\circ}$, depending on the curvature of the fiber bundle. These empirically optimized thresholds reflect a priori anatomical knowledge. For example, the FA values in gray matter are lower than in white matter because of a more randomized fiber orientation within an image voxel and a much lower degree of myelination. Because the absolute values depend on the chosen MRI sequence, the spatial resolution, the filtering, and the diffusion model, the minimum acceptable FA value for fiber tracking in white matter is commonly determined with respect to the actual application and in many studies close to 0.15 as used here. Similar arguments apply to the limiting values for the curvature of specific fiber tracts. For example, when reconstructing the pyramidal fiber bundles, the known anatomical pathway suggested a restriction to a relatively low curvature of only $20^{\circ}$, while the cingulum bundle required a maximum curvature of $60^{\circ}$.

Tracking of distinct fiber bundles was accomplished with use of different strategies depending on the fiber composition, curvature, and size. In all cases the definition of regions-of-interest (ROIs) - on color-coded maps of the main diffusion direction - was entirely based on existing anatomical knowledge with reference to classical textbooks $[7,8]$. In order to obtain a most faithful virtual dissection of white matter fasciculi, only minor adjustments were necessary to match individual conditions. Thus, beside the definition of a seed (and target) ROI and the choice of the FA and curvature thresholds, no further editing was employed. 
A single seed ROI was used for anatomically welldescribed fibers where no other neighboring fasciculi hinder the virtual tract to travel along the correct anatomical pathway. A typical example without false-positive deviations is the cingulum bundle which is situated above the body of the corpus callosum beneath the gray matter of the cingulum. In more critical cases, a second region helped to properly direct the virtual tract reconstruction or to exclude divergencies. The NOT operation for the second ROI was employed to remove tract reconstructions that share image voxels with the desired tract but do not coincide with its classical definition, a situation that for example applied to the superior longitudinal fasciculus and fornix. Alternatively, the $A N D$ option for a seed and target ROI, also termed region-to-region tracking [4,5], served to minimize false-positive fiber reconstructions that result from fasciculi running in close proximity to one another $[7,8]$. The $A N D$ strategy allows for a separation of such fibers if they become distinct in other more remote brain regions. For example, the technique was used to reconstruct the pyramidal fiber bundles by placing a seed ROI near the internal capsule and a target ROI close to the motor and somatosensory cortex, respectively.

\section{RESULTS}

\section{Association Fiber Pathways}

Association fibers travel in discrete fiber bundles to other cortical areas within the same hemisphere. Respective fiber bundles either remain in the adjacent gyrus or the same lobe, or travel deeper into the white matter of the same hemisphere. Figs. $(\mathbf{1}, \mathbf{2})$ summarize three-dimensional views of the main association fibers identified in an individual rhesus monkey by DTI. The color code refers to the local fiber direction, or more precisely to the local mean diffusion direction (red = left-right, green $=$ anterior-posterior, blue $=$ superior-inferior). The complementary views are superimposed onto the individual monkey's high-resolution 3D MRI anatomy.

\section{Superior Longitudinal Fasciculus}

The superior longitudinal fasciculus (SLF) shown in the top row of Fig. (1) is one of the large fiber systems that connect the parietal lobe with the frontal lobe. In humans the SLF and the arcuate fasciculus have long been considered synonymous and both names have been used interchangeably in different anatomical studies $[15,16]$. In line with the results of Schmahmann and colleagues [17] in monkeys we found a separated tract with fibers traveling between the inferior parietal regions and the frontal lobe areas, which is different to the SLF described in human DTI studies [16]. Already in 1984, Petrides and Pandya [18] separated the SLF in the monkey brain into three components, which vary with respect to position and termination areas in the parietal lobe. In our observations, the fibers lie caudally deep in the white matter of the parietal lobe and extend rostrally in a prominent fan-shaped manner, connecting the inferior parietal lobe with the posterior prefrontal areas as assumed for the SLF II. Therefore, we assigned the tract shown in Fig. (1) at least in part to the SLF II.

\section{Uncinate Fasciculus}

The uncinate fasciculus (UF) was one of the five long association fasciculi discussed by Dejerine [6] - also called the hooked fasciculus. The UF is the most rostral temporal fiber bundle, linking the anterior temporal lobe with the orbital and polar frontal cortex in a bidirectional way [8]. As shown in the middle row of Fig. (1), the UF takes indeed the shape of an anteromedial hook to terminate in the temporal lobe.

\section{Inferior Longitudinal Fasciculus}

Several lines of evidence indicate that the inferior longitudinal fasciculus (ILF) shown in the bottom of Fig. (1) runs in the white matter of the parietal, occipital, and temporal lobe. The ILF conveys the information in a bidirectional manner between the occipital lobe and the temporal lobe. Earlier and recent data show that the ILF is almost vertically oriented in the parietal-occipital region. As reviewed by Schmahmann and Pandya [8] the use of gross dissections and histochemical studies as well as lesion studies has led to a persistent uncertainty regarding the existence of the ILF with researchers confusing the sagittal stratum, the optic radiation, and the ILF. Isotope studies in monkeys dispelled the uncertainty of the ILF pathway [8]. In close agreement with this work, we could clearly identify the ILF by fiber tractography in an individual macaque without overlap with the optic radiation, that is without arborizations in the lateral geniculate nucleus. We therefore support the recent terminology.

\section{Fronto-Occipital Fasciculus}

This association fasciculus links occipital regions with frontal brain areas and is shown in the top part of Fig. (2). The fronto-occipital fasciculus (FOF) touches laterally the corona radiatus and medially the corpus callosum. Some fibers pass through the SLF before entering the FOF, where they travel rostrally into the frontal lobe. Schmahmann and Pandya [8] described two different sets of fiber bundles moving along the rostro-caudal direction. They clearly separate the FOF and the Muratoff bundle. In contrast to the FOF, the Muratoff bundle is a distinct fiber tract that carries fibers from the occipital, parietal, temporal, and frontal lobe and terminates in the caudate nucleus (not shown). The Muratoff bundle is therefore strictly a corticostriatal bundle. As shown in Fig. (2) the large fiber bundle identified by DTI travels along the rostral-caudal direction, connecting the occipital lobe and parts of the frontal lobe without terminations in the caudate nucleus. Although the existence of the FOF was the subject of several discussions, the general pathway of the fiber tract seems to be in line with the anatomical and histochemical studies.

\section{U-Fiber}

Local association fibers (U-fibers) originate from a given cortical area to terminate in an adjacent gyrus, transmitting a feedback from the neighborhood area [8]. An example is shown in the bottom of Fig. (2). These selected U-shaped fibers connect regions of the primary motor cortex with parts of the primary sensory cortex.

\section{Limbic System}

The upper part of Fig. (3) shows the fornix (left) and the cingulum bundle (right) using the same directional color code as for Figs. $(\mathbf{1}, \mathbf{2})$. To better distinguish between both structures, the lower part of Fig. (3) depicts two different 

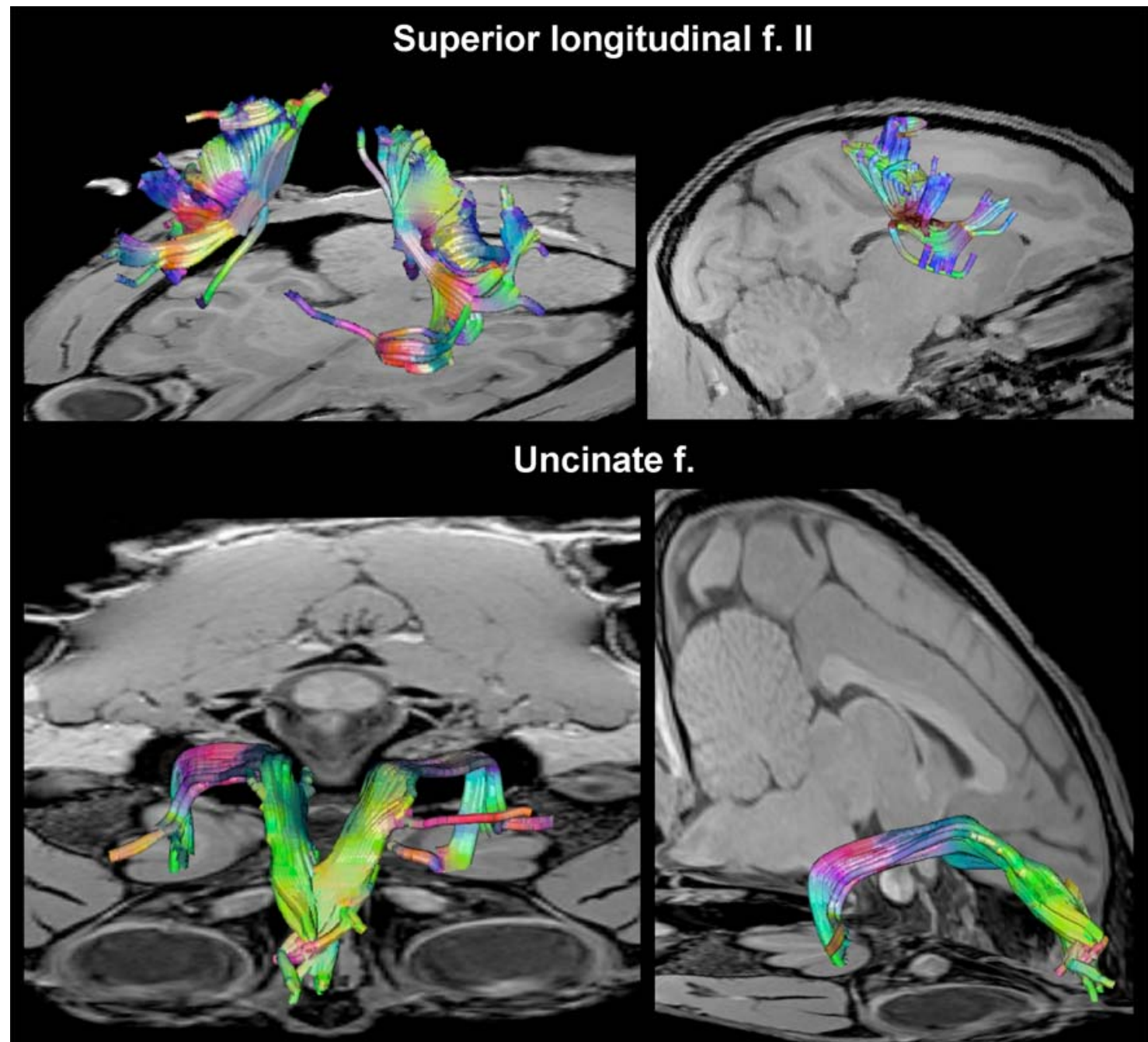

\section{Inferior longitudinal f.}

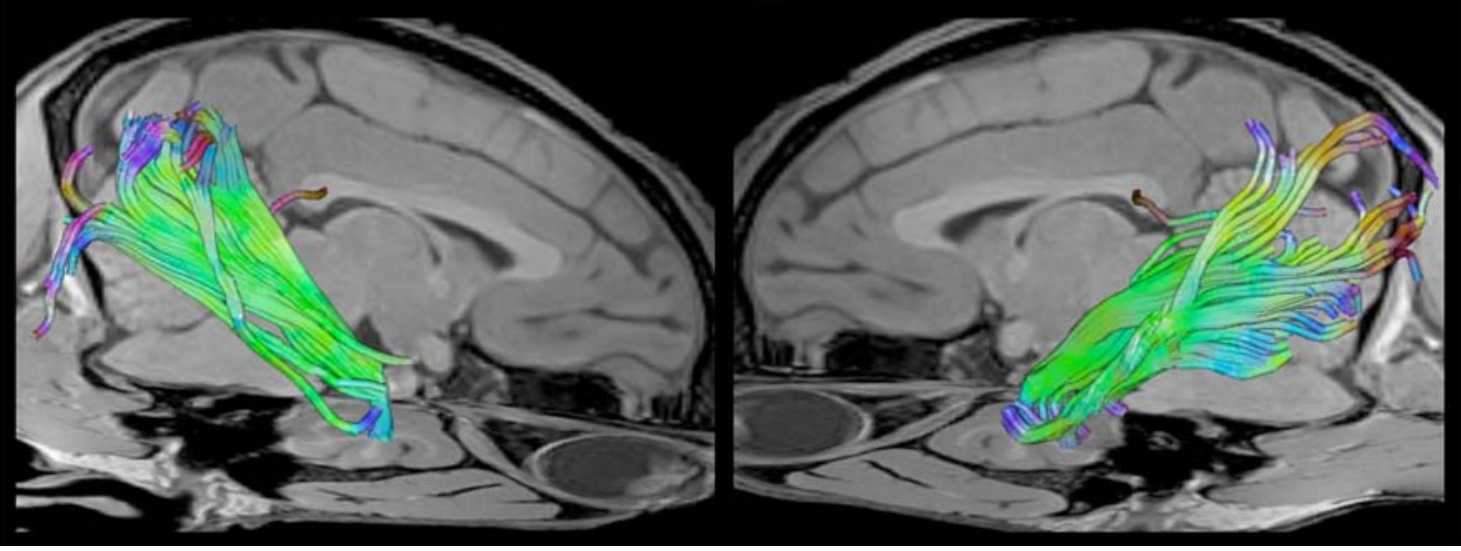

Fig. (1). Association fibers. Three-dimensional views overlaid onto individual anatomical images of the monkey brain with colors corresponding to the local mean diffusion direction: red $=$ left-right, green $=$ anterior-posterior, blue $=$ superior-inferior. $($ Top) Superior longitudinal fasciculus II in (left) an oblique frontal view of the right and left hemisphere and (right) a sagittal view of the right hemisphere. (Middle) Uncinate fasciculus along rostro-caudal and mediolateral directions in (left) a frontal view and (right) an oblique sagittal view. (Bottom) Inferior longitudinal fasciculus in sagittal views of the (left) right and (right) left hemisphere.

views of these bundles with distinct uniform colors (red: fornix, blue: cingulum).

The cingulum bundle contains fibers of different lengths. The longest bundle runs from the parahippocampal gyrus to the frontal lobe [19]. The fibers run from the parahippocampal gyrus posteriorly to revolve around the splenium by almost 180 degrees. They extend above the corpus callosum and stretch around the cingulate gyrus. Shorter fibers leave 


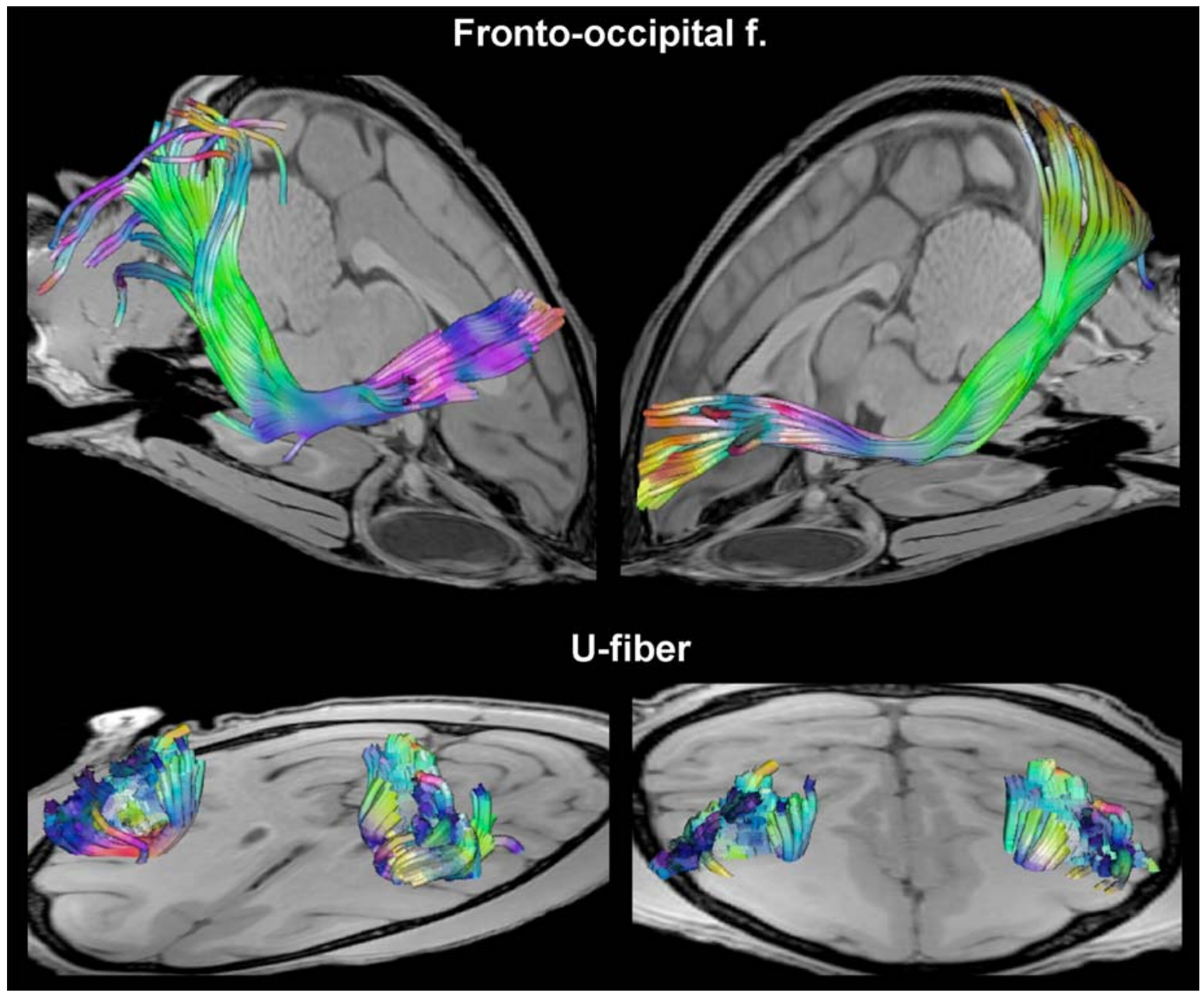

Fig. (2). Association fibers (parameters as in Fig. (1)). (Top) Fronto-ocipital fasciculus in oblique sagittal views of the (left) right and (right) left hemisphere. (Bottom) U-fibers connecting parts of the somatosensory cortex with motor areas in frontal views.

the tract along its length connecting to the medial frontal and parietal lobe.

The fornix is a limbic structure connecting the hippocampus with the mamillary bodies [6]. Fibers arise from the hippocampal area within each hemisphere and run in a curved path beneath the splenium of the corpus callosum into the hypothalamus as shown in Fig. (3). By DTI we could clearly identify the body of the fornix, running below the body of the corpus callosum toward the anterior commissure. The body is divided into a symmetric right and left part, belonging to each hemisphere.

\section{Projection Fibers}

All projection fiber bundles consist of efferent and afferent fibers connecting various parts of the cortex with the lower parts of the brain and the medulla. Selected pyramidal tracts are shown in Fig. (4).

Pyramidal tract neurons were identified in precentral motor cortex areas and in the postcentral cortex of the monkey brain. By definition, the pyramidal tract consists of all fibers crossing longitudinally the pyramid of the medulla oblongata, regardless of their site of origin [20,21]. The fibers exhibit a fan-like arrangement, which opens toward the cortex and closes when approaching the internal capsule.
Pyramidal tract neurons in the primary somatosensory cortex of the monkey show that these neurons have properties in common with pyramidal tract neurons of primary motor cortex. Therefore, the somatosensory cortex has a direct role in the control of movement [22].

As demonstrated in Fig. (4) we could indeed identify large fiber bundles that take course from the primary motor cortex and the somatosensory cortex through the internal capsule. Because DTI is blind to the direction of the tracts (afferent or efferent), these tracts include both ascending fibers mainly terminating in the sensory area and descending fibers mainly originating from the motor cortex.

\section{Commissures}

Commisural fibers refer to all nerve fibers in the brain that cross the midline and connect the symmetrical halves of the central nervous system. The corpus callosum (CC) shown in Fig. (5) is the largest commissure that connects most of the neocortical areas. It presents with a topographical representation of the different cortical areas as established in different placental mammals (reviewed in [22]). In macaque the topography reflects the wide diversity of fiber calibers [12,23-25]. DTI confirms that many callosal connections are homotopic and connect equivalent regions between the two hemispheres (for details see [12]). 


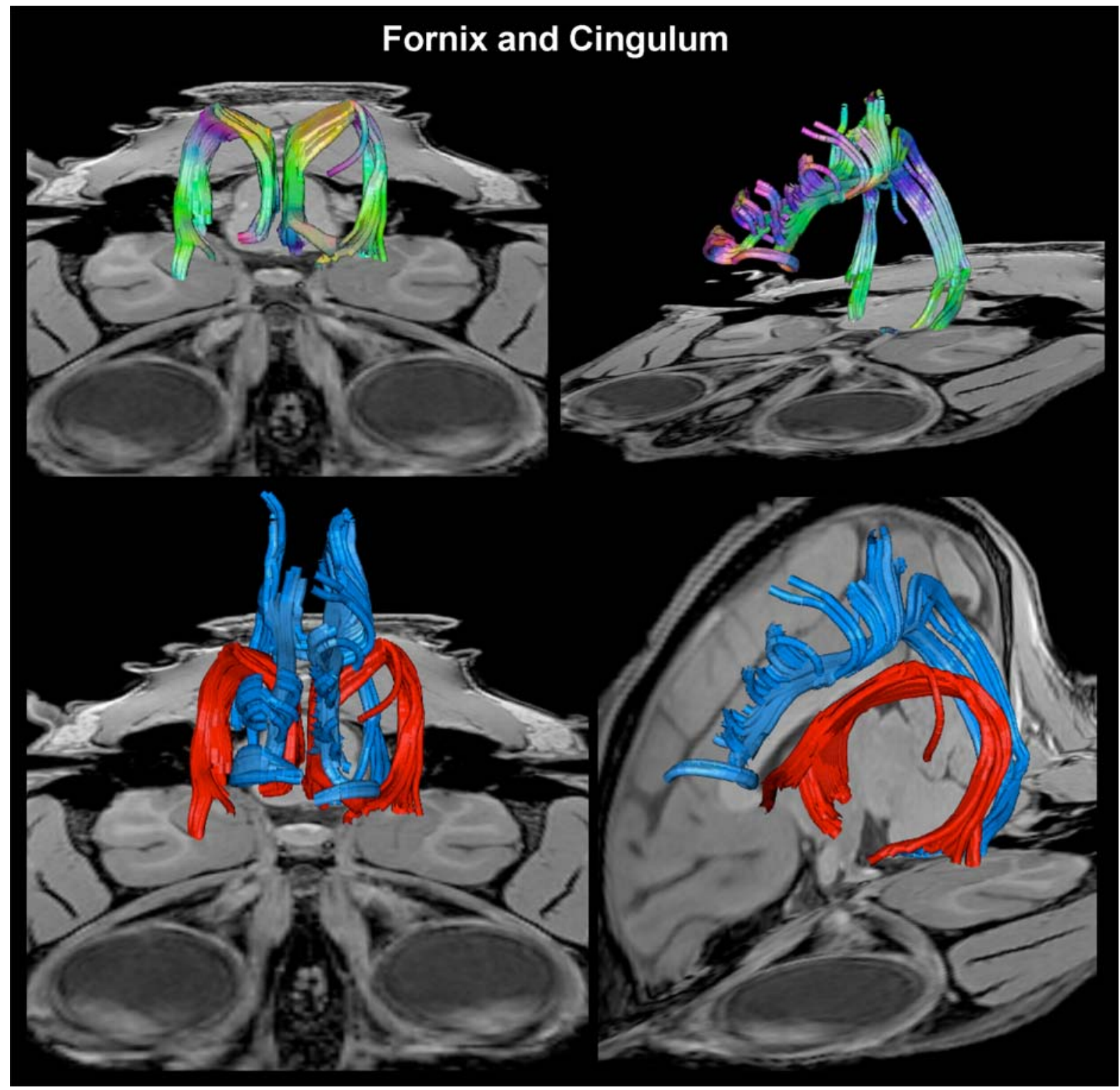

Fig. (3). Fibers of the limbic system. (Top left) Fornix in a frontal view and (right) cingulum in an oblique frontal view (color codes for direction as in Fig. (1)). (Bottom) Separation of fornix (coded in red) and cingulum (blue) in (left) a frontal view and (right) an oblique sagittal view of the left hemisphere.

\section{Optic System}

The visual pathway extends from anterior to posterior and comprises the optic nerve, chiasm, optic tract, and optic radiation to terminate in the primary visual cortex. As shown in Fig. (6) all components of this pathway are identified by DTI of the rhesus monkey brain.

Optic nerves are found in all vertebrates with eyes and are composed of axons with retinal ganglion cells. In the chiasm of the mammalian brain only the nasal retinal fibers cross to the opposite hemisphere. The respective optic tracts terminate in the lateral geniculate nucleus (LGN), where the fibers are arranged in a precise retinotopic manner as shown in histochemical studies. The LGN is a major relay structure transmitting the optic impulse via the optic radiation to the visual cortex [26]. Because of methodological limitations it is not possible to follow the path of the nasal-retinal fibers in the chiasm, so that these fibers are missing in the optic nerve. Furthermore, the approach is unable to demonstrate that the LGN is a relay station of the visual system, because the DTI-based fiber tracts pass through its gray matter to terminate in the visual cortex. Nevertheless, the identification of the entire visual pathway demonstrates a large degree of hemispheric symmetry with fibers that clearly follow the route as revealed in recent anatomical and histochemical studies.

\section{Cerebellar System}

The cerebellum has numerous connections to other brain areas. The human cerebellum contains about half of the total number of neurons in the brain and, in terms of structure, reveals an impressive and regular order. The pontine nuclei and the inferior olive are important relay stations, transmitting information from the brain via the brainstem to the cerebellum and vice versa [27]. As seen in Fig. (7), DTI dis- 


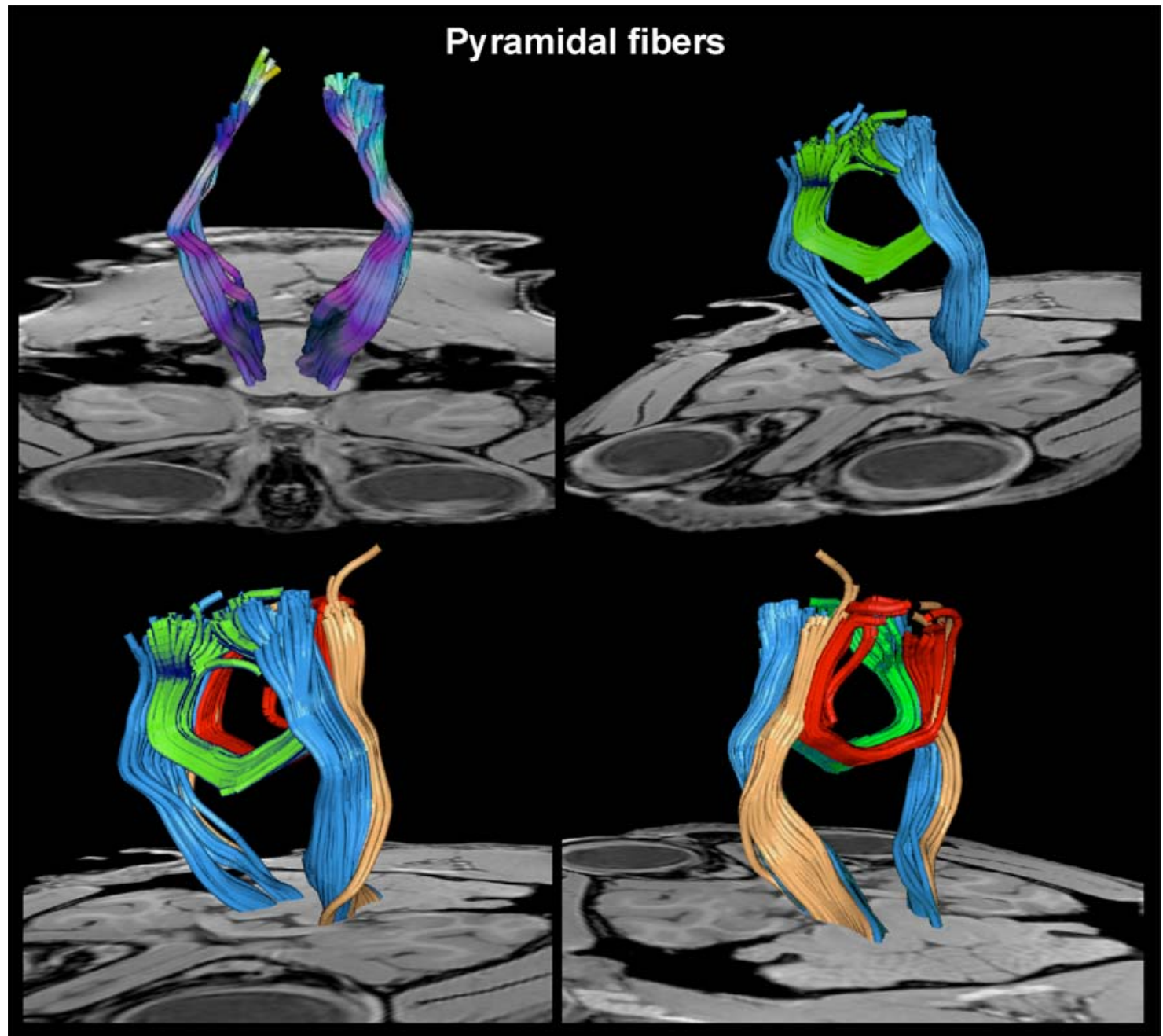

Fig. (4). Pyramidal and callosal fibers. (Top left) Pyramidal motor fibers in a frontal view (color codes for direction as in Fig. (1)). (Top right) Separation of pyramidal motor fibers (coded in light blue) and transcallosal motor fibers (green). (Bottom) Pyramidal motor fibers (light blue) and callosal motor (green) together with pyramidal somatosensory fibers (orange) and transcallosal somatosensory fibers (red) in (left) an oblique frontal and (right) rear view.

criminated between three different fiber pathways innervating cerebellar structures that we assume to belong to the middle peduncular fiber system, inferior peduncular fiber bundles, and the superior peduncular fibers.
The brainstem consists of the medulla oblongata, pons, and the midbrain, containing numerous ascending and descending fibers as well as fibers that originate from and terminate in it. The ventral part comprises transverse fiber sys-

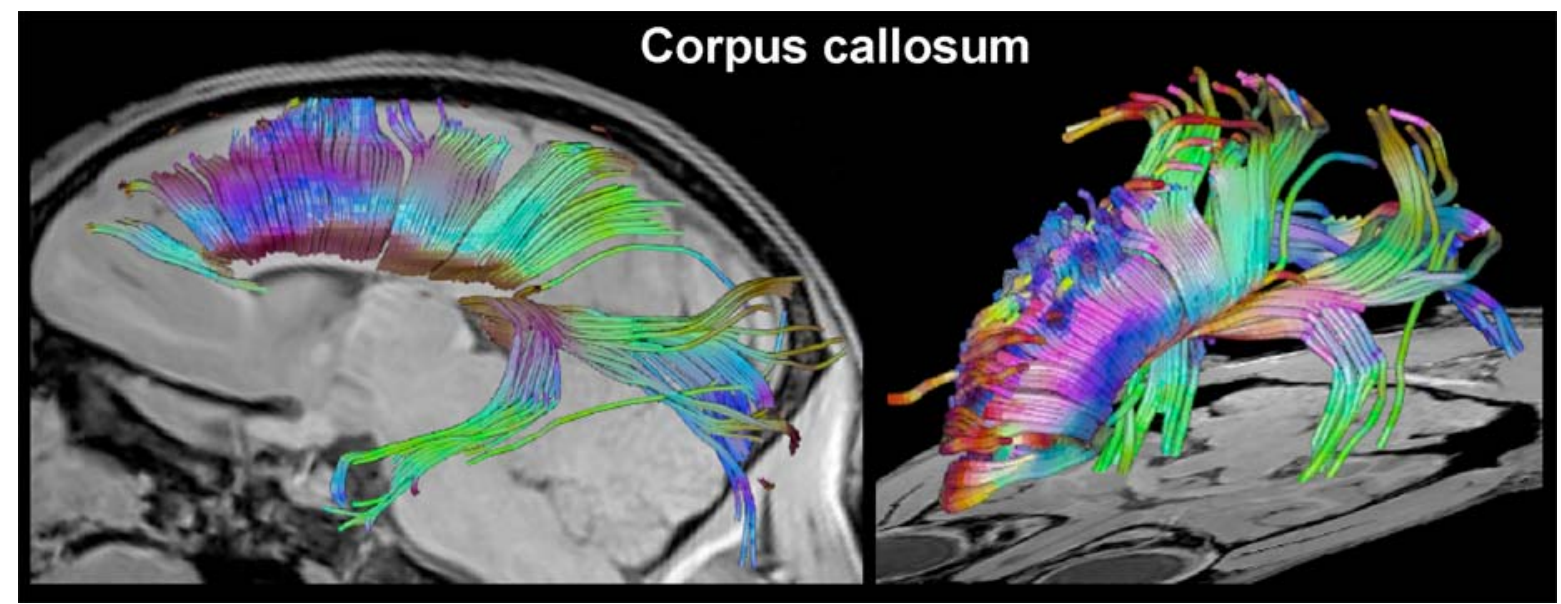

Fig. (5). Transcallosal fibers. (Left) Sagittal view and (right) oblique frontal view (color codes for direction as in Fig. (1)). 


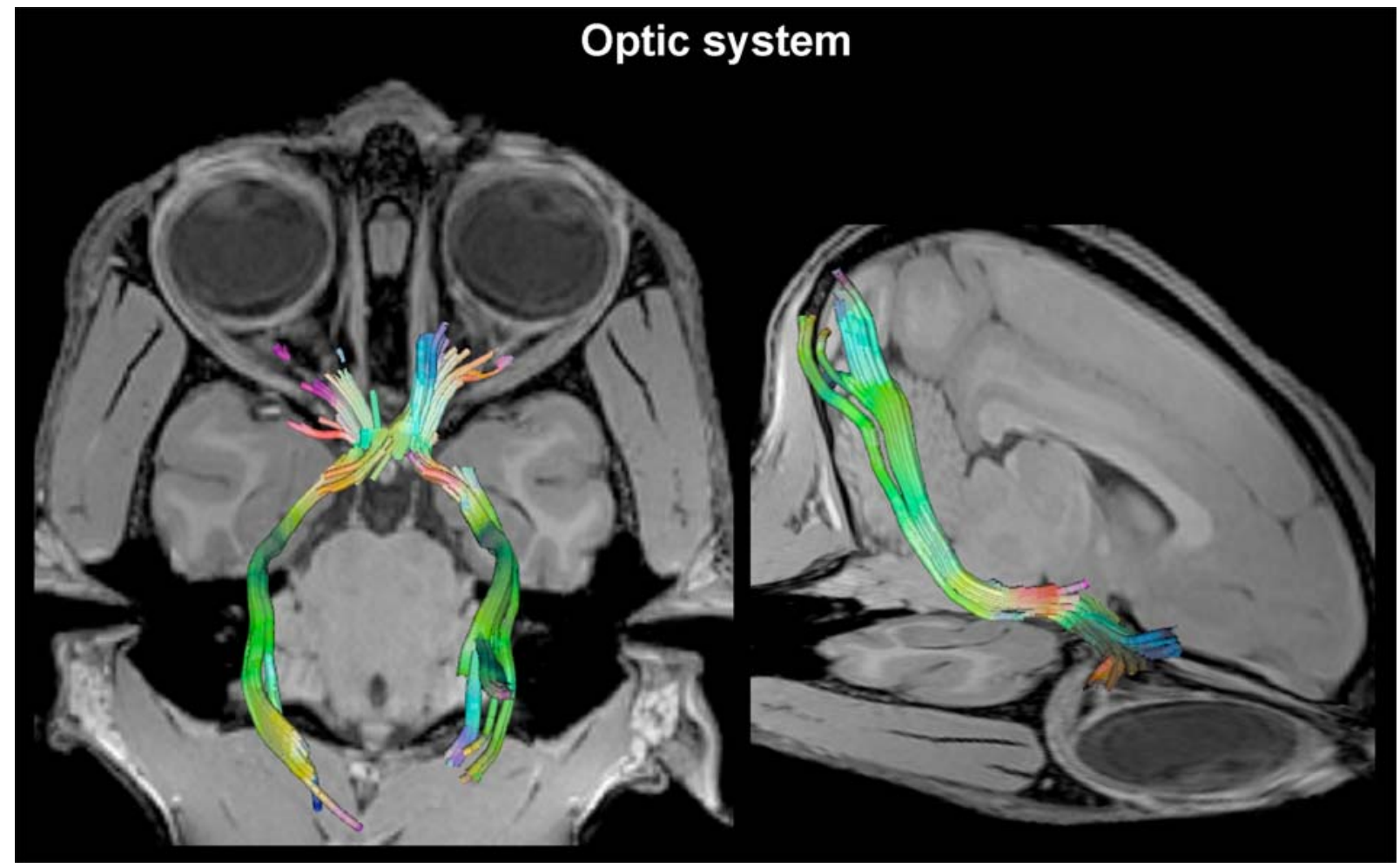

Fig. (6). Fibers of the optic system. Optic nerve, optic tract, and optic radiation in (left) a top view and (right) an oblique sagittal view of the right hemisphere (color codes for direction as in Fig. (1)).

tems connecting the pons with the cerebellum, via the middle cerebellar peduncle fiber system. The superior cerebellar peduncle is the major output pathway of the cerebellum. Most of the efferent fibers originate within the dentate nucleus which in turn projects to various midbrain structures. The inferior cerebellar peduncle carries many types of input and output fibers that are mainly concerned with integrating sensory input with motor functions such as balance and posture maintenance $[26,27]$.

\section{DISCUSSION}

Recent technical developments using DTI-based fiber tractography have made it possible to visualize fiber bundles in the human brain in vivo [16,28]. Validation of the result-

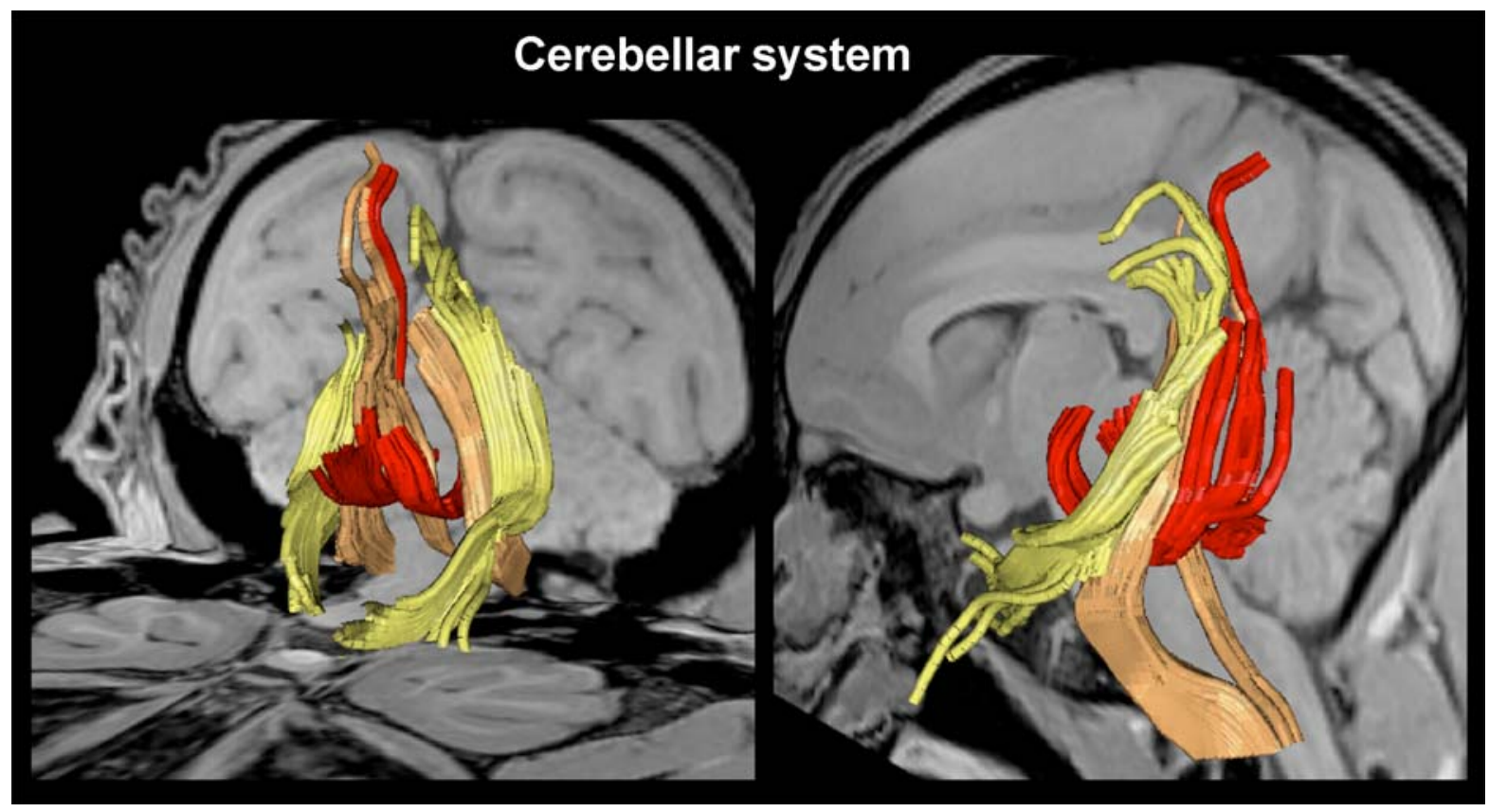

Fig. (7). Fibers of the cerebellar system. Middle cerebellar peduncle fiber system (coded in yellow), superior cerebellar peduncle system (red), and inferior cerebellar fiber system (orange) in (left) a frontal view and (right) an oblique sagittal view of the right hemisphere. 
ing fiber tracts has relied upon findings from pioneering investigators who mainly used human lesion studies for tract identification [6]. Nevertheless, the anatomy of fiber tracts in the human brain is not as well established as the literature suggests which often results in inaccuracies and terminological confusions (e.g., see [8]). Moreover, because most of the detailed anatomical data has been obtained in non-human primates, we should be cautious in assigning DTI-based fiber reconstructions in the human brain. In this study, we therefore investigated fiber pathways in the rhesus macaque brain in vivo and compared them with fiber pathways revealed by established histochemical tract tracing methods in the same species. Thus, we were able to fully explore the potential of diffusion-weighted MRI and fiber tractography to identify several fiber pathways throughout a whole macaque brain, first and foremost with a DTI technique not suffering from geometric image distortions due to susceptibility differences $[10,11]$.

The present work extends and underlines other in vivo MRI studies of the rhesus monkey brain, which - apart from an earlier feasibility study [29] - mainly focused on the somatosensory and motor system using similar tract tracing techniques [30] or on prefrontal areas using probabilistic fiber tractography [31]. Although the latter approach yields a comprehensive list of all possible connections, it does not offer a straightforward mechanism for identifying individual fiber pathways as attempted here.

More recent in vitro studies dealt with high-resolution fiber tracking in fixated monkey brains in order to facilitate a more direct validation of DTI-based fiber tracts against traditional histochemical assessments [17,32]. The advantage of post mortem DTI is the possibility of using complex diffusion encoding strategies with long measuring times such as diffusion spectrum imaging (DSI). Schmahmann and colleagues [17] identified 10 long association tracts and compared them with the white matter tracts revealed by autoradiographic histologic tract tracing [8]. Despite general agreement and some very impressive results, the technique hampers from the need for a very large number of gradient directions (e.g., 515 directions were considered necessary to obtain data of good quality) that lead to very long acquisition times (e.g., imaging times added up to 25 hours) precluding studies of anesthetized animals or human patients. Noteworthy, even under these favorable post mortem conditions, "kissing fibers" [33] could not be resolved as good as expected - or in a manner comparable to classical histochemical studies. Moreover, because the animals were part of a stroke study and all had an ischemic lesion in one hemisphere, fiber tractography was restricted to the contralesional hemisphere.

DTI-based fiber tractography is a visualization technique generating lines that reflect the anatomy of axonal trajectories. The main advantage of the MRI approach is its noninvasiveness which allows for a three-dimensional reconstruction of various fiber bundles with reference to the individual high-resolution anatomy, whether of a human or monkey brain. In this respect, we were able to confirm most of the results from the aforementioned post mortem DSI study with a much simpler and faster diffusion technique in vivo (24 gradient directions, 28 min measuring time). By means of in vivo DTI we could visualize various association fibers, projection fibers, callosal fibers, and three cerebellar fiber bundles as well as the fibers of the entire optic system. In particular, the insensitivity of the used STEAM DTI technique to tissue susceptibility differences and related magnetic field inhomogeneities allowed us to identify even frontal optical fibers in areas, where conventional DTI studies based on echo-planar imaging usually fail to provide any meaningful data or suffer from severe geometric distortions. Nevertheless, DTI-based fiber tractography is still an emerging technology with many unsolved problems and challenges. For example, complications arise for situations where two fiber bundles cross in one image voxel because the simple tensor model (visualized as a corresponding diffusion ellipsoid) would result in a flat disk representing the two dominating directions. In this case the assumption of a single preferred orientation would cause the tracking algorithm to fail. Similarly, nerve fibers that run in parallel but belong to separate tracts may be difficult to distinguish based on the directional information alone, so that reconstructed nerve fibers may "jump over" and continue along the false tract. Another important practical limitation is the achievable resolution and signal-to-noise ratio (per available measuring time in vivo). Both parameters may critically affect the determination of the major eigenvector of the diffusion tensor and therefore lead to cumulative directional errors in the estimated fiber tracts. Taken together, the reconstruction of nerve fiber tracts from DTI data deserves some caution. The present work therefore contains tracts of only the most prominent white matter fasciculi in the rhesus monkey brain that have previously been described using histological techniques.

A major issue in basic and clinical neuroscience is the correlation of white matter structure and function. Several lines of evidence indicate that a damaged or disrupted connectivity of a particular fiber bundle due to a focal brain lesion is indeed associated with a specified functional deficit, a behavioral change, or clinical symptom. Examples comprise the fornix, cingulum, uncinate, corpus callosum, the frontal and temporal white matter, and the cerebellar peduncle [27,34-37]. Various diseases or syndromes such as schizophrenia have long been suggested to be related to abnormal fiber connectivity [38]. Further preliminary DTI studies of white matter pathology already extend to aging, aphasia, dementia, multiple sclerosis, HIV, and various forms of leukodystrophies (reviewed by Malloy et al. [39]). In this respect, a simple and powerful imaging technique for mapping the white matter architecture that underlies the large-scale neuronal networks responsible for our cognitive functions would be of great scientific and clinical help. As shown here for the monkey brain, DTI-based fiber tractography can detect different fiber pathways in vivo that are in line with histochemically proven neuroanatomical insights.

\section{ACKNOWLEDGEMENTS}

We gratefully acknowledge the technical assistance of Dr. Klaus-Dietmar Merboldt and Dr. Roland Tammer.

\section{REFERENCES}

[1] Wouterlood FG, Vinkenoog M, van den Oever M. Tracing tools to resolve neural circuits. Network 2002; 13: 327-42. 
[2] Basser PJ, Pierpaoli C. Microstructural and physiological features of tissues elucidated by quantitative diffusion-tensor MRI. J Magn Reson B 1996; 111: 209-219.

[3] Mori S, Crain BJ, Chacko P, van Zijl PC. Three-dimensional tracking of axonal projections in the brain by magnetic resonance imaging. Ann Neurol 1999; 45: 265-269.

[4] Mori S, Kaufmann WE, Davatzikos C, et al. Imaging cortical association tracts in the human brain using diffusion-tensor-based axonal tracking. Magn Reson Med 2002; 2: 215-23.

[5] Hofer S, Frahm J. Topography of the human corpus callosum revisited - comprehensive fiber tractography using diffusion tensor magnetic resonance imaging. Neuroimage 2006; 32: 989-994.

[6] Dejerine J. Anatomie des Centres Nerveux vol. 2, 1895; Rueff et Cie, Paris.

[7] Ludwig E, Klingler J. Atlas Cerebri Humani, 1956; S Karger, Basel.

[8] Schmahmann JD, Pandya DN. Fiber Pathways of the Brain. Oxford University Press, 2006.

[9] Boretius S, Würfel J, Zipp F, Frahm J, Michaelis T. High-field diffusion tensor imaging of mouse brain in vivo using single-shot STEAM MRI. J Neurosci Methods 2007; 161: 112-117.

[10] Nolte UG, Finsterbusch J, Frahm J. Rapid isotropic diffusion mapping without susceptibility artifacts. Whole brain studies using diffusion-weighted single-shot STEAM MR imaging. Magn Reson Med 2000; 44: 731-736.

[11] Rieseberg S, Merboldt K-D, Küntzel M, Frahm J. Diffusion tensor imaging using partial Fourier STEAM MRI with projection onto convex subsets reconstruction. Magn Reson Med 2005; 54: 486490.

[12] Hofer S, Merboldt K-D, Tammer R, Frahm J. Rhesus monkey and human share a similar topography of the corpus callosum as revealed by diffusion tensor MRI in vivo. Cereb Cortex 2007; in press Aug 20 [Epub ahead of print]

[13] Weinstein D, Kindlmann G, Lundberg E. "Tensorlines: Advectiondiffusion based propagation through diffusion tensor fields," In IEEE Visualization, 1999; 249-253.

[14] Küntzel M. Parallele Datenakquisition zur Beschleunigung diffusions- gewichteter Kernspintomographie mit stimulierten Echos [Parallel data acquisition for diffusion-weighted MRI using stimulated echoes]. Dissertation, Georg-August-Universität, Göttingen 2006. Online access of the University library: http: //webdoc.sub. gwdg.de/diss/2007/kuentzel/kuentzel.pdf.

[15] Klingler J, Goor P. The connections of the amygdale and of the anterior temporal cortex in the human brain. J Comp Neurol 1960; 115: 333-369.

[16] Catani M, Howard RJ, Pajevic S, Jones DK. Virtual in vivo interactive dissection of white matter fasciculi in the human brain. Neuroimage 2002; 17: 77-94.

[17] Schmahmann JD, Pandya DN, Wang R, et al. Association fibre pathways of the brain: parallel observations from diffusion spectrum imaging and autoradiography. Brain 2007; 130: 630-53.

[18] Petrides M, Pandya DN. Projections to the frontal cortex from the posterior parietal region in the rhesus monkey. J Comp Neurol 1984; 228: 105-16.

[19] Baleydier C, Mauguiere F. The duality of the cingulate gyrus in monkey. Neuroanatomical study and functional hypothesis. Brain 1980; 103: 525-54.

[20] Fromm C, Wise SP, Evarts EV. Sensory response properties of pyramidal tract neurons in the precentral motor cortex and postcentral gyrus of the rhesus monkey. Exp Brain Res 1984; 54: 177-85.
[21] Ebeling U, Reulen HJ. Subcortical topography and proportions of the pyramidal tract. Acta Neurochir (Wien) 1992; 118: 164-71.

[22] Fromm C, Evarts EV. Pyramidal tract neurons in somatosensory cortex: central and peripheral inputs during voluntary movement. Brain Res 1982; 22: 186-91.

[23] Aboitiz F, Montiel J. One hundred million years of interhemispheric communication: the history of the corpus callosum. Braz Med Biol Res 2003; 36: 409-20.

[24] Pandya DN, Karol EA, Heilbronn D. The topographical distribution of interhemispheric projections in the corpus callosum of the rhesus monkey. Brain Res 1971; 32: 31-43.

[25] LaMantia AS, Rakic P. Cytological and quantitative characteristics of four cerebral commissures in the rhesus monkey. J Comp Neurol 1990; 291: 520-537.

[26] Tamraz JC, Comair Y. Atlas of regional anatomy of the brain using MRI with functional correlations. New York, Springer, 2006.

[27] Ramnani N. The primate cortico-cerebellar system: anatomy and function. Nat Rev Neurosci 2006; 7: 511-22.

[28] Wakana S, Jiang H, Nagae-Poetscher LM, van Zijl PC, Mori S. Fiber tract-based atlas of human white matter anatomy. Radiology 2004; 230: 77-87.

[29] Parker GJM, Stephan KE, Barker GJ, et al. Initial demonstration of in vivo tracing of axonal projections in the macaque brain and comparison with the human brain using diffusion tensor imaging and fast marching tractography. Neuroimage 2002; 15: 797-809.

[30] Dauguet J, Peled S, Berezovskii V, et al. Comparison of fiber tracts derived from in-vivo DTI tractography with 3D histological neural tract tracer reconstruction on a macaque brain. Neuroimage 2007; 15: 530-538.

[31] Croxson PL, Johansen-Berg H, Behrens TE, et al. Quantitative investigation of connections of the prefrontal cortex in the human and macaque using probabilistic diffusion tractography. J Neurosci 2005; 28: 8854-66.

[32] D'Arceuil HE, Westmoreland S, de Crespigny AJ. An approach to high resolution diffusion tensor imaging in fixed primate brain. Neuroimage 2007; 35: 553-65.

[33] Basser PJ, Pajevic S, Pierpaoli C, Duda J, Aldroubi A. In vivo fiber tractography using DT-MRI data. Magn Reson Med 2000; 44: 625 32 .

[34] Gaffan D, Parker A, Easton A. Dense amnesia in the monkey after transection of fornix, amygdala and anterior temporal stem. Neuropsychologia 2001; 39: 51-70.

[35] Kubicki M, McCarley R, Westin CF, et al. A review of diffusion tensor imaging studies in schizophrenia. J Psychiatr Res 2007; 41: 15-30.

[36] Highley JR, Walker MA, Esiri MM, Crow TJ, Harrison PJ. Asymmetry of the uncinate fasciculus: a post-mortem study of normal subjects and patients with schizophrenia. Cereb Cortex 2002; 12: 1218-24

[37] Rodrigo S, Oppenheim C, Chassoux F, et al. Uncinate fasciculus fiber tracking in mesial temporal lobe epilepsy. Initial findings Eur Radiol 2007; 12 [Epub ahead of print].

[38] Kubicki M, Park H, Westin CF, et al. DTI and MTR abnormalities in schizophrenia: analysis of white matter integrity. Neuroimage 2005; 26: 1109-18.

[39] Malloy P, Correia S, Stebbins G, Laidlaw DH. Neuroimaging of white matter in aging and dementia. Clin Neuropsychol 2007; 21: 73-109. 